\title{
Avaliação de desempenho de ativímetros para medição dos radiofármacos PET baseados em ${ }^{18} \mathrm{~F}$
}

\author{
M.C.F. Fragoso ${ }^{a}$, M.L. Oliveira ${ }^{\mathrm{a}}$, F.R.A. Lima ${ }^{\mathrm{a}}$ \\ ${ }^{a}$ Centro Regional de Ciências Nucleares do Nordeste (CRCN-NE) / Comissão Nacional de Energia Nuclear \\ (CNEN) Av. Professor Luiz Freire, $n^{\circ} 200$ - Cidade Universitária - Recife - PE CEP:50740-545 \\ mariacc05@gmail.com
}

\begin{abstract}
RESUMO
Testes de proficiência são ferramentas essenciais para avaliar a confiabilidade e a exatidão das medições. Neste trabalho, testes de proficiência (Z-score, exatidão e desvio relativo) foram aplicados para avaliar o desempenho dos ativímetros utilizados na Divisão de Produção de Radiofármacos do Centro Regional de Ciências Nucelares do Nordeste (CRCN-NE/CNEN). Os resultados foram avaliados em conformidade à norma ISO/IEC 43-1. Adicionalmente, fatores de correção para diferentes geometrias de medição foram determinados experimentalmente para estes equipamentos.
\end{abstract}

Palavras-chave: Ativímetros; ${ }^{18} \mathrm{~F}$; testes de proficiência; fatores de correção.

\begin{abstract}
Proficiency test is an essential tool to assess the reliability and accuracy of measurements. In this work, proficiency tests (Z-score, accuracy and relative deviation) were applied to evaluate the performance of the activimeters used at Radiopharmaceuticals Production Division of the Regional Center for Nuclear Sciences of the Northeast (CRCNNE/CNEN). The results were evaluated in compliance with ISO/IEC Guide 43-1. Additionally, correction factors for different measurement geometries were determined experimentally for those devices.
\end{abstract}

Keywords: Activimeter; ${ }^{18} \mathrm{~F}$; proficiency tests; correction factors. 


\section{INTRODUÇÃO}

A tomografia por emissão de pósitrons (PET - Positron Emission Tomography) é uma das mais importantes técnicas de imagem molecular disponíveis na atualidade. O radiofármaco PET mais utilizado é o fluordeoxiglicose $\left({ }^{18} \mathrm{~F}\right.$-FDG). Na rotina clínica, antes que o radiofármaco seja administrado ao paciente é necessário que se conheça com exatidão a sua atividade para garantir imagens com qualidade suficiente para o diagnóstico médico. Em PET, esta medição exata torna-se mais importante uma vez que a relação entre a concentração de atividade no tumor (obtida a partir da imagem) e a atividade total administrada fornece uma informação importante sobre a resposta do indivíduo ao tratamento, podendo levar a alterações na conduta médica (KINAHAN; FLETCHER, 2010). Desta forma, torna-se imprescindível garantir o bom desempenho dos sistemas de medição deste radionuclídeo (ativímetros) nos centros de produção e nos serviços de medicina nuclear (SMN).

A medida da atividade fornecida pelo ativímetro pode ser influenciada por vários fatores, que incluem o próprio funcionamento do equipamento. Desta forma, é indispensável que testes de controle de qualidade sejam realizados e que o comportamento do equipamento seja avaliado ao longo do tempo.

Outro aspecto importante a ser considerado está relacionado à influência da geometria de medição nas respostas obtidas pelos ativímetros. Estudos realizados acerca do controle da qualidade dos ativímetros sugerem testes (posição, volume e recipiente da amostra) para assegurar correções adequadas na geometria de medição das amostras radioativas, cuja importância e influência dependem significativamente do tipo de radiação emitida (ZIMMERMAN, 2006; CESSNA et al., 2008; CORREIA et al., 2012).

O propósito deste trabalho foi avaliar o desempenho dos ativímetros pertencentes à Divisão de Produção de Radiofármacos (DIPRA) do Centro Regional de Ciências Nucelares do Nordeste (CRCN-NE/CNEN) por meio de testes de proficiência (Z-score, exatidão e desvio relativo), baseados nos critérios de aceitação da norma ISO/IEC 43-1 (ISO, 1997). Adicionalmente, foi verificado o efeito de amostras produzidas em diferentes geometrias nas respostas obtidas por estes equipamentos. 


\section{MATERIAIS E MÉTODOS}

O Laboratório de Medidas de Atividade de Radionuclídeos da DIPRA/CRCN-NE possui três ativímetros comerciais (tabela 1). Estes equipamentos foram submetidos aos testes de controle da qualidade estabelecidos por normas nacionais e internacionais (CNEN, 2013; LNHB, 2006; NPL, 2006), com o intuito de assegurar a confiabilidade das medições de radiofármacos.

Tabela 1: Características dos ativímetros utilizados neste trabalho.

\begin{tabular}{lccc}
\hline Ativímetros & A & B & C \\
\hline Fabricante & Capintec & Biodex & PTW \\
Modelo & CRC-15R & ATOMLAB 500 & T12004 \\
Gás & Argônio & Argônio & Argônio \\
Altura do poço (cm) & 25,4 & 26 & 32,9 \\
Diâmetro do poço (cm) & 6,1 & 6,4 & 6,15 \\
Blindagem (mm chumbo) & 3,2 & 6,3 & 3,8 \\
Exatidão & $\pm 2 \%$ & $\pm 3 \%$ & $\pm 5,5 \%$ \\
\hline
\end{tabular}

Para obtenção das soluções de referência dos radiofármacos PET baseados em ${ }^{18} \mathrm{~F}$, foi utilizado o método padrão-amostra, sendo obtidas seis diferentes soluções mantendo-se a geometria (geometria frasco padrão CRCN) (tabela 2).

Tabela 2: Características das soluções de referência de ${ }^{18} \mathrm{~F}$.

\begin{tabular}{lcc}
\hline & $\begin{array}{c}\text { Concentração de Atividade } \\
(\mathbf{k B q} / \mathbf{g})\end{array}$ & Data de referência* \\
\hline Rodada 1 & $225,39 \pm 0,54$ & $26 / 07 / 2013$ \\
Rodada 2 & $103,82 \pm 0,99$ & $26 / 07 / 2013$ \\
Rodada 3 & $108,56 \pm 0,74$ & $27 / 07 / 2013$ \\
Rodada 4 & $95,07 \pm 0,53$ & $02 / 10 / 2013$ \\
Rodada 5 & $153,9 \pm 1,8$ & $25 / 10 / 2013$ \\
Rodada 6 & $111,91 \pm 0,57$ & $07 / 11 / 2013$ \\
\hline
\end{tabular}

*Corrigida para $12 \mathrm{~h} 00$.

As medidas de atividade foram realizadas em cada um dos ativímetros listados na tabela 1 , conforme procedimento padrão adotado pelo laboratório (10 medidas com intervalo de 10 s entre 
elas), obtendo-se a média das leituras e o desvio padrão. Em seguida, os resultados obtidos foram avaliados segundo os critérios de aceitação estabelecidos pela ISO/IEC 43-1.

O Z-score foi obtido por meio da seguinte equação:

$$
Z_{\text {scor } B}=\frac{\left(V_{E x p}-V_{R e f}\right)}{\sigma}
$$

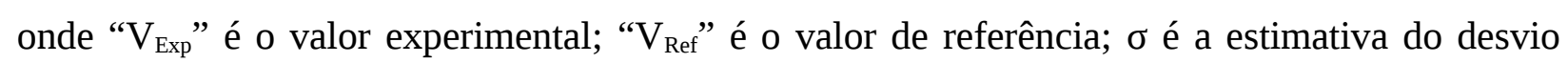

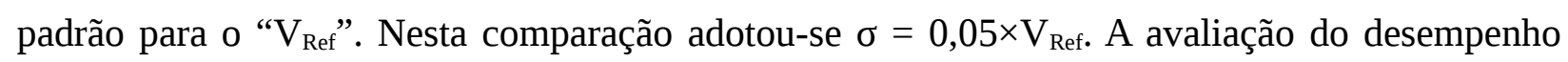
segue o seguinte critério: $|\mathrm{z}| \leq 2$ indica desempenho satisfatório; se $2<|\mathrm{z}|<3$, o desempenho é questionável; e quando $|\mathrm{z}| \geq 3$ o desempenho é considerado insatisfatório.

A exatidão ( $\left.\mathrm{U}_{\text {score }}\right)$ foi avaliada mediante a seguinte expressão:

$$
U_{\text {scor } \theta}=\left|\frac{V_{R e f}-V_{E x p}}{k \times \sqrt{u_{R E f}^{2}-u_{E x p}^{2}}}\right|
$$

onde " $u_{R e f}$ " e " $u_{\operatorname{Exp}}$ " são as incertezas padrões do valor de referência e do valor experimental, respectivamente; $\mathrm{k}$ é o fator de abrangência desejado (neste trabalho foi adotado $\mathrm{k}=2$ para um nível de confiança de 95\%). O resultado será considerado aceitável se $\left|U_{\text {score }}\right| \leq 1$.

O desvio relativo foi determinado pela razão entre a diferença do valor obtido no processo de medição e o valor de referência da grandeza medida pelo valor de referência, dado em valor percentual. O limite de aceitação para este teste consiste em $\pm 10 \%$.

Para verificar a influência da mudança de frasco na resposta do ativímetro foram utilizados os fracos mais comumente utilizados nos SMN em Recife, PE (o frasco CRCN e o frasco IPEN). Adicionalmente, foram testados os frascos padrões de laboratórios de calibração internacionais (P6 e 10R) (BAKER, 2005) (figura 1). 
Figura 1: Frascos de vidro utilizados para a verificação dos efeitos ocasionados na resposta do ativímetro devido à alteração da geometria de medição.

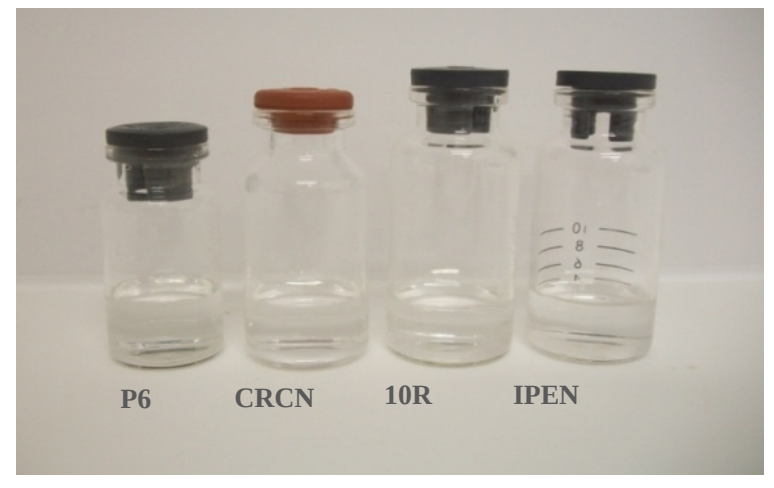

Fonte: Os autores.

Os fatores de correção para geometria de medição em relação ao frasco padrão CRCN foram determinados por meio da razão entre o valor da atividade medida na geometria padrão e o valor da atividade medida nos demais frascos.

\section{RESULTADOS E DISCUSSÃO}

Os resultados obtidos para o teste Z-score e exatidão são apresentados na figura 2. Verificou-se que $83 \%$ dos resultados avaliados mediante os critérios do teste Z-score, indicaram desempenho "Satisfatórios” e apenas 3\% foram considerados “Questionáveis”. No que se refere ao teste de exatidão, apenas 67\% dos resultados foram “Aceitáveis”. 
Figura 2: Resultados do teste Z-score (a) e exatidão (b) obtidos na avaliação de desempenho dos ativímetros A (marca Capintec), B (marca Biodex) e C (marca PTW) disponíveis na DIPRA/CRCN-NE.

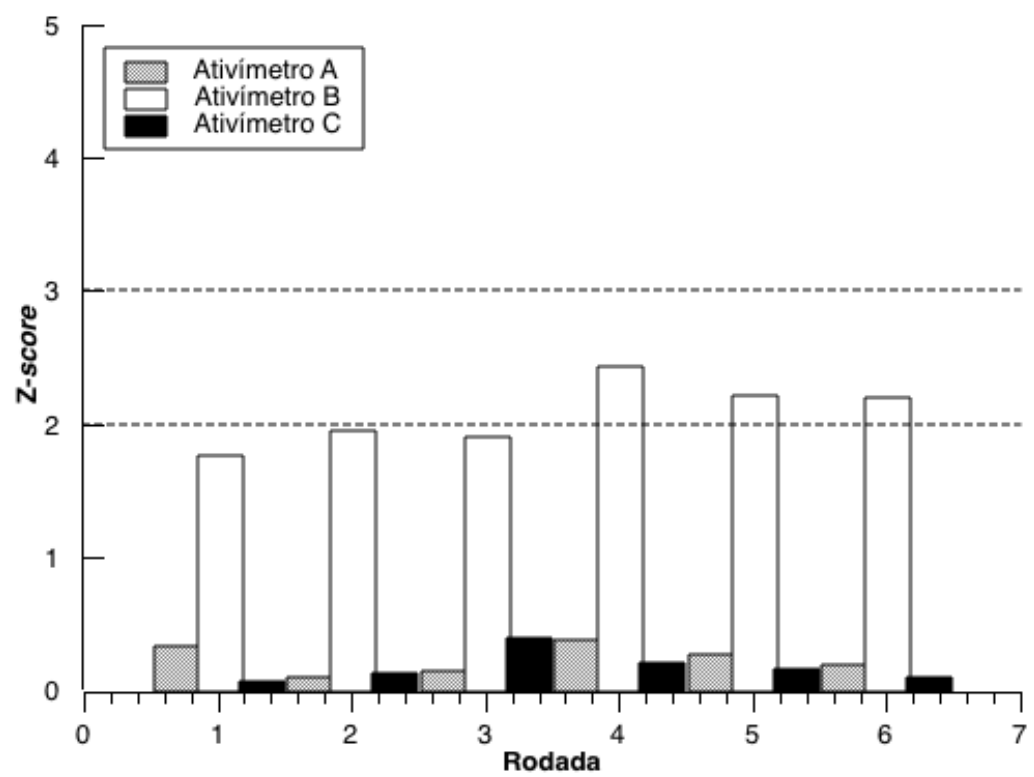

(a)

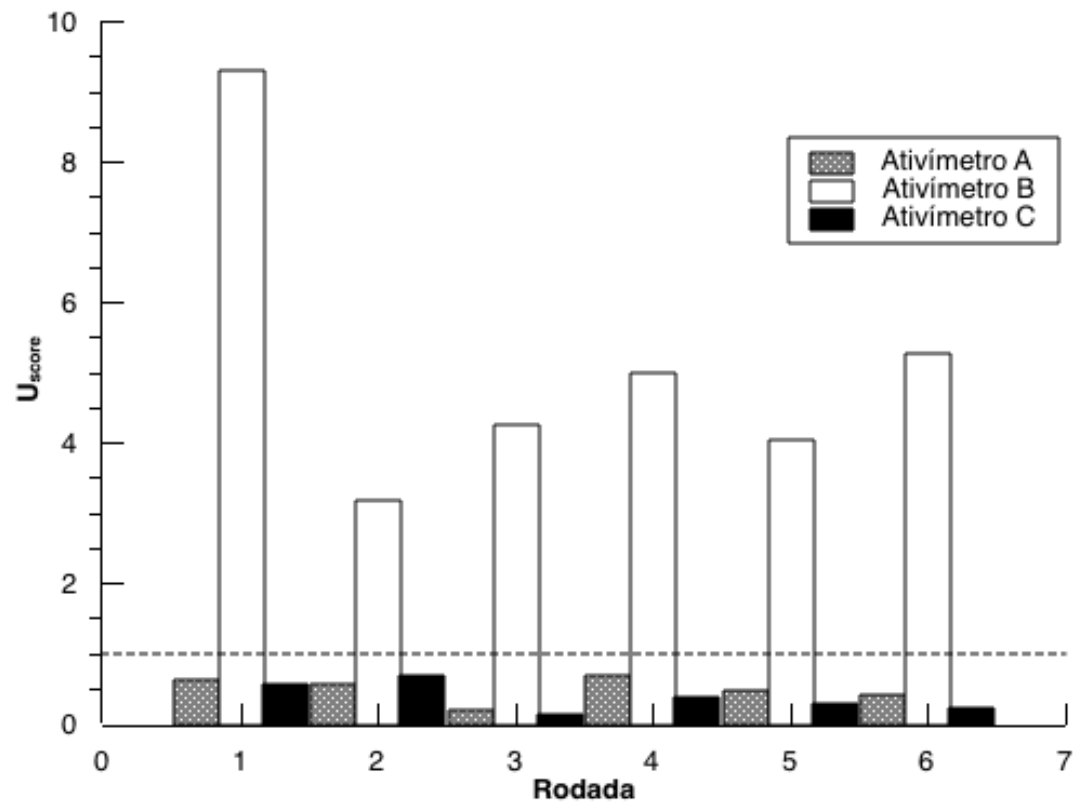

(b) 
Fonte: Os autores

Desvios relativos inferiores a 2\% foram observados para os ativímetros A e C e acima da ordem de 10\% para o ativímetro B. Segundo os critérios de avaliação estabelecidos na norma ISO/IEC 43-1, apenas o ativímetro B apresentou resultados acima dos limites recomendados para os testes Z-score, exatidão e desvio relativo. Diante disso, recomenda-se uma nova calibração do equipamento para a energia do ${ }^{18} \mathrm{~F}$.

Os fatores de correção para diferentes tipos de recipientes (P6, 10R e IPEN) em relação ao frasco CRCN foram experimentalmente determinados, conforme apresentados na tabela 2. Os resultados demonstraram a variação na leitura do ativímetro em relação à geometria de medição da amostra. Desta forma, os fatores de correção devem ser utilizados caso a amostra de ${ }^{18} \mathrm{~F}$ a ser medida esteja acondicionada em um desses frascos.

Tabela 2: Fatores de correção para diferentes tipos de recipientes em relação ao frasco CRCN.

\begin{tabular}{lccc}
\hline Frasco & Ativímetro A & Ativímetro B & Ativímetro C \\
\hline P6 & $0,889 \pm 0,004$ & $0,995 \pm 0,006$ & $0,978 \pm 0,003$ \\
10R & $0,911 \pm 0,004$ & $1,011 \pm 0,009$ & $1,008 \pm 0,003$ \\
IPEN & $0,935 \pm 0,005$ & $1,022 \pm 0,009$ & $1,019 \pm 0,004$ \\
\hline
\end{tabular}

\section{CONCLUSÕES}

Com base nos resultados experimentais obtidos neste trabalho, verificou-se um desempenho satisfatório dos ativímetros das marcas Capintec e PTW. No entanto, o ativímetro da marca Biodex apresentou resultados acima dos limites recomendados pela norma ISO/IEC 43-1. Diante disso, recomenda-se uma nova calibração do equipamento para a energia do ${ }^{18} \mathrm{~F}$.

Fatores de correção para os frascos padrões de laboratórios de calibração nacionais (frasco padrão IPEN) e internacionais (frascos P6 e 10R) foram determinados experimentalmente, com o intuito de assegurar a confiabilidade na exatidão das medições de atividade. 


\section{AGRADECIMENTOS}

Os autores agradecem ao Conselho Nacional de Desenvolvimento Científico e Tecnológico (CNPq), ao Instituto Nacional de Ciência e Tecnologia em Metrologia das Radiações em Medicina (INCT) e à Comissão Nacional de Energia Nuclear (CNEN).

\section{REFERÊNCIAS}

BAKER, M. Calibration of the NPL secondary standard radionuclide calibrator for the new 10R Schott, Type 1+ vials. Appl Radiat Isotopes, v. 63, p. 71-77, 2005.

CESSNA, J.T.; SCHULT, M.K.; LESLI, T.; BORES, N. Radionuclide calibrator measurements of ${ }^{18} \mathrm{~F}$ in a $3 \mathrm{ml}$ plastic syringe. Appl Radiat Isotopes, v. 66, p. 988-993, 2008.

\section{CNEN - COMISSÃO NACIONAL DE ENERGIA NUCLEAR. Requisitos de Segurança e} Proteção Radiológica para Serviços de Medicina Nuclear - CNEN-NN- 3.05. Rio de Janeiro, 2013.

CORREIA, A. R.; IWAHARA, A.; TAUHATA, L.; REZENDE, E.A.; CHAVES, T.O.; DE OLIVEIRA, A.E.; OLIVEIRA, E.M. Volume corrections factors in the measurement of ${ }^{99 \mathrm{~m}} \mathrm{Tc}$ and ${ }^{123} \mathrm{I}$ activities in radionuclide calibrators. Radiol Bras, v. 45, p. 93-97, 2012.

ISO - International Organization for Standar-dization. ISO/IEC Guide 43-1. Proficiency testing by inter-laboratory comparisons - Part 1: Development and operation of proficiency testing schemes. Geneva, 1997.

KINAHAN, P.E.; FLETCHER, J.W. PET/CT Standardized uptake values (SUVs) in clinical practice assessing response to therapy. Semin Ultrasound CT MR, v. 31, p. 496-505, 2010. 
LNHB - LABORATOIRE NATIONAL HENRI BECQUEREL. Guide d'utilisation et de contrôle qualité des activimètres. Societé française de radiopharmacie. França: LNHB, 2006.

NPL - NATIONAL PHYSICAL LABORATORY. Protocol for Establishing and Maintaining the Calibration of Medical Radionuclide Calibrators and their Quality Control. Measurement Good Practice Guide No 93. Londres: NPL, 2006.

ZIMMERMAN, B.E. Radionuclide metrology in the life sciences: Recent advances and future trends. Appl Radiat Isotopes, v. 64, p.1351-1359, 2006. 